\section{Laterality and visual persistence: Still a two-sided issue}

\section{STEPHEN A. WURST and GERALD M. LONG Villanova University, Villanova, Pennsylvania}

In a recent article, Di Lollo (1981) attempted to resolve "ostensible inconsistencies" in the literature concerning hemispheric differences in visual persistence. Specifically, he obtained no significant differences in performance for stimuli presented to the left visual field (LVF) and the right visual field (RVF) on a persistence task. With this task, fifteen dots in a $4 \times 4$ array were presented sequentially over a plotting interval of 50,100 , or $150 \mathrm{msec}$, and the observer reported which position in the array did not contain a dot. From this null effect, Di Lollo concluded that the issue of laterality and visual persistence was resolved, because with this task "successful performance rests unambiguously upon visible persistence alone. Thus, from this perspective, the absence of hemispheric asymmetries in duration of visible persistence can be explained on the joint grounds that visible persistence is produced at an initial stage of processing, and that early stages of information processing are handled similarly and with equal efficiency by both hemispheres"' (p. 25). It is the contention of the present note, however, that such a generalized conclusion is not justified and can be questioned on several grounds. In addition to the problems Di Lollo failed to address, evidence reported since the appearance of his article presents further difficulties for his conclusion. It is believed that both methodological and theoretical considerations can be cited to show that Di Lollo may have overstated his case and that his conclusion is premature. The present paper focuses on the most obvious of these points.

\section{Methodological Considerations}

Di Lollo's major argument is that his task unambiguously measures "visible persistence" and that two previous studies claiming laterality in persistence do not. However, findings in laterality studies in general are notoriously task dependent (see White, 1972). To conclude from one task under one set of stimulus conditions that persistence is not lateralized is presumptuous at best. As noted recently by Sergent (1982a), visual laterality studies must take into account a myriad of stimulus factors: "There are practical considerations that must not be overlooked

The author's mailing address is: Department of Psychology, Villanova University, Villanova, Pennsylvania 19085. in future research. Any factor susceptible of influencing the quality of stimulus representation has to be controlled; due to the properties of the visual system, one cannot select the duration of exposure, the size of the stimulus, its visual angle from fixation, or the delay between target and test presentation ... without examining how they affect the characteristics of the representation achieved in the brain. Similarly, the requirements of the tasks to be performed must be analyzed"' (p. 269).

Therefore, a discussion of the stimulus conditions and the task employed by Di Lollo is in order.

Stimulus duration. The exposure duration of the stimulus has been found to be an important determinant of laterality differences. Bryden (1965), for example, found that very small changes in duration produced significantly different results on a tachistoscopic recognition task. More recently, Sergent (1982b) showed that on a face-identification task, an LVF superiority occurs with a 40-msec duration, but an RVF superiority occurs with a 200 -msec duration. Although such a pronounced duration effect is not universally reported in laterality research (e.g, Polzella, DaPolito, \& Hinsman, 1977), it still remains possible that a duration-dependent laterality effect in persistence can exist. In the Di Lollo (1981) study, in which each successive stimulus was presented for an extremely minute $1 \mu \mathrm{sec}$, these potential effects are not considered. Hence, the effect of duration on the laterality of persistence is an unanswered question that must yet be dealt with empirically.

Type of persistence. Another problem with Di Lollo's (1981) conclusion concerning that apparent hemispheric symmetry of visible persistence is that different tasks can measure different "types" of persistence. In the current persistence literature, a distinction has been made between "Type I" and "Type II" persistence (Bowling \& Lovegrove, 1982; Hawkins \& Shulman, 1979; Long, 1979, 1982; Long \& McCarthy, 1982a). Type I persistence appears to be related to the observer's latency for responding to the physical offset of this stimulus, that is, to the minimal detectable difference between the actual stimulus and the decaying trace. Type II persistence, on the other hand, refers to the total duration of the rapidly fading representation of the stimulus. Although in other studies Di Lollo (e.g., Di Lollo \& Woods, 1981) has suggested that his task assesses Type II persistence (but see Long, 1982), he does not raise this issue in the symmetry-of-persistence study. It is possible that visual-field differences could occur with persistence tasks that assess the other type of persistence. At the very least, the question remains unanswered.

Adequacy of task. Di Lollo (1981) believes that the inconsistencies in the lateralization-of-persistence 
literature are due to the inadequacy of the tasks used by Polzella, DaPolito, and Hinsman (1977) and Erwin and Nebes (Note 1). Di Lollo argues that the tasks used by these investigators were heavily influenced by higher levels of processing and that these effects were not adequately taken into account. However, in two earlier studies (not cited by Di Lollo) dealing with laterality and persistence, the effects of higher level processing were carefully considered. Cohen (1976) employed seven different conditions in order to isolate the effects of (1) the length of the "icon," (2) the rate of encoding from the icon, and (3) attentional differences. By separating these other influences, she concluded that stimuli persisted longer in the LVF than in the RVF. Also, Marzi, DiStefano, Tassinari, and Crea (1979) performed a series of experiments with a partial-report task in order to identify any higher processing effects that may also have influenced the persistence estimates. By comparing the results of experiments using spatial (e.g., rectangles) and verbal stimuli, Marzi et al. concluded that the laterality effects they did find were most likely due to higher levels of processing and that no visual-field differences in persistence exist. Although Di Lollo would claim that these tasks measure "schematic" (i.e., nonvisual) rather than visible persistence, this is a controversial issue in the persistence literature at the present time (e.g., Long, 1982; Long \& Beaton, 1982; Long \& McCarthy, 1982b).

Nature of the stimulus. Other methodological considerations that Di Lollo failed to address are stimulus factors such as the verbal/nonverbal nature of the stimulus and the complexity of the stimulus. This is especially important in view of the fact that some laterality researchers contend that the nature of the stimulus itself is critical for the detection of laterality differences (Simion, Bagnara, Bisiachi, Roncato, \& Umilta, 1980). Very simply, Di Lollo employed stimuli that could be considered spatial (dots in a $4 \times 4$ matrix), but he did not consider the possibility that the persistence for other kinds of stimuli could yield different results.

The complexity of the stimulus may also represent a seriously overlooked factor. In both the laterality and the persistence literature, the complexity level of the stimulus has been shown to have significant effects on results. For example, in the laterality literature, Fontenot (1973) found an LVF superiority for high-complexity figures but no laterality effects for low-complexity figures on a multiplechoice stimulus-recognition task. Similarly, Umilta, Bagnara, and Simion (1978) found an LVF superiority for very complex figures when observers were instructed to distinguish whether two figures were the same or different. In the persistence literature, Wurst and Long (Note 2) and Long and Wurst (Note 3) have reported complexity differences on a common visual persistence task. Wurst and Long (Note 2) found that as operationally defined complexity of solid, random-shape figures increased, perceived duration of persistence decreased, due to the contribution of purely sensory factors. However, when outline figures of the same shapes were used, perceived persistence increased with increasing complexity, suggesting the role of postsensory processes (Long \& Wurst, Note 3). Hence, how critical the simplicity of the stimuli in Di Lollo's (1981) study are for the null effect he obtains is unknown.

\section{Theoretical Considerations}

In addition to the several methodological issues enumerated above, recent research raises some questions about Di Lollo's conclusions on a more theoretical level. For example, Sergent (1982b) conducted a series of experiments to test the hypothesis that laterality differences may be the result of the relative ability of the left hemisphere (LH) and the right hemisphere $(\mathbf{R H})$ to handle the spatial-frequency components of the stimulus. The results of these experiments, in which she presented faces at different durations and had her observers respond whether the face was male or female, supported her hypothesis. From these results she concluded that "the main suggestion is that the LH preferentially operates on, and produces, high-resolution information, whereas the RH electively deals with, and yields low-resolution information"' (p. 459). As she also notes, the neural channels that deal with high- versus low-frequency information are the so-called sustained and transient channels, respectively, that have been identified by numerous physiological and psychophysical studies (e.g., Fukuda \& Stone, 1974; Harwerth, Boltz, \& Smith, 1980; Regan, 1982; Williamson, Kaufman, \& Brenner, 1978). Hence, Sergent (1982b) proposes an imbalance in the distribution of these channels across the two hemispheres.

Interestingly, Di Lollo and Woods (1981) argued that visual persistence effects are directly dependent upon this sustained and transient channel activity. Consistent with the work of Bowling and Lovegrove (1980) and Meyer and Maguire (1977, 1981), Di Lollo and Woods found that high spatial frequencies induced longer persistence than low spatial frequencies. They concluded that "the compelling implication of these results is that the reduction of high spatial frequencies in the display is responsible for the progressive decrement in duration of visible persistence .... It must be assumed that duration of visible persistence is determined, at least in part, by the duration of activity within the sustained channels"' (p. 766).

It seems, then, that Di Lollo accepts the argument that persistence is dependent to some extent upon sustained and transient channel activity. If Sergent's (1982b) recent proposal that laterality effects are due to an asymmetry across the hemisphere in the dis- 
tribution of sustained and transient channels is indeed correct, Di Lollo may well find himself on both sides of the issue concerning laterality and persistence.

\section{Conclusions}

The major contention of this note is that the issue of whether or not visual persistence is lateralized is still very much unresolved. One particular task with one limited set of stimulus conditions cannot provide the answer for an issue as complex as the lateralization of visual persistence. What kind of persistence is involved on the task chosen? What levels of luminance and duration are employed? What kind of stimuli are presented? And there are other serious questions not raised in the above sections. What about sex differences, a currently controversial topic in the laterality research? What about the nature of the dependent variable (e.g., verbal response vs. manual reaction time)? The answer to all these questions must be investigated before the issue of the asymmetry in persistence can be determined.

As a brief demonstration of the relevance of the above questions to the issue at hand, consider the results shown in Figure 1. These are from a reanalysis of a portion of the data reported by Long and Beaton (1982) in a recent article. The task was a standard partial-report persistence task in which the observer reports which of eight letters in a 50-msec circular array of letters is indicated by a probe that points at one of the letter positions. The task involved persistence because the probe marker follows the offset of the letter array by some interval (ISI). The Long and Beaton data have been reanalyzed in terms of visual field effects, as shown in Figure 1. Note the significant interaction in the figure $[F(7,28)=2.81, p<$ $.05]$. At the longer ISIs, there is a significant RVF superiority in report of the letters. Although this might be interpreted as reflecting greater visual persistence in the LH or, as Di Lollo (1981) might argue, greater schematic persistence in the $\mathrm{LH}$, the point we wish to stress here is that these results were obtained only with the $3.0 \mathrm{fL}\left(3.43 \mathrm{~cd} / \mathrm{m}^{2}\right)$ target luminance level. For the other luminances examined $(1.0,15$, and $30 \mathrm{fL}$ ), no effect of visual field was obtained for the very same observers. Again, the point is not that the results in Figure 1 support an RVF superiority of visual persistence, but rather that it is important to examine a multitude of potentially interactive factors when dealing with an area this complex before "resolving" an issue.

Therefore, there cannot be a blanket rejection of laterality in visual persistence yet. It may be that Di Lollo is indeed correct in stating that there are no laterality effects in visual persistence. Moreover, evidence of asymmetry in persistence would not even be readily interpretable. Current models of visual persistence attribute the phenomenon to everything from

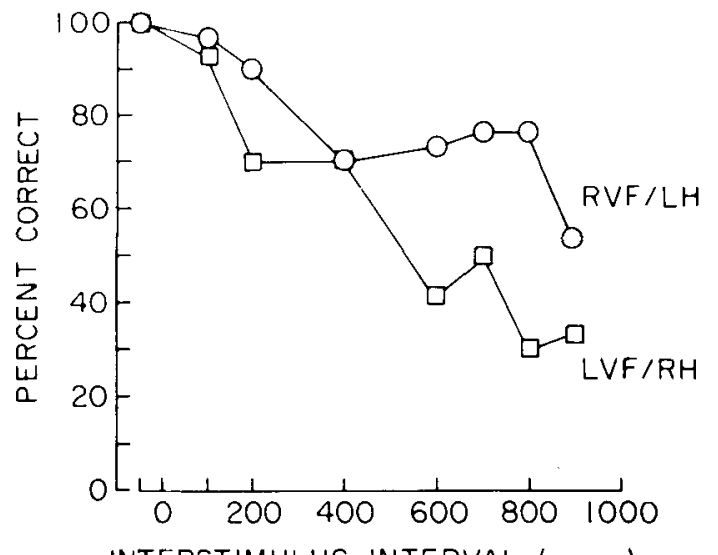

Figure 1. Mean percent correct as a function of interstimulus interval for letters presented in the left visual field (LVF/RH) and right visual field (RVF/LH) on a partial-report task. (Date from Long \& Beaton, 1982)

sluggish photoreceptor activity to the time it takes higher centers to extract the stimulus information, and from relative sustained channel activity to the existence of a cognitive buffer (cf. Bowling \& Lovegrove, 1980; Coltheart, 1980; Di Lollo, 1980; Long, 1980). The argument being made in this note is simply that more empirical evidence is needed before the issue of laterality in persistence is settled.

\section{REFERENCE NOTES}

1. Erwin, D. E., \& Nebes, R. D. Right hemispheric involvement in the functional properties of visual persistence. Paper presented at the annual meeting of the Eastern Psychological Association, New York, April 1976.

2. Wurst, S. A., \& Long, G. M. Stimulus complexity and perceived duration. Paper presented at the annual meeting of the Eastern Psychological Association, Baltimore, April 1982.

3. Long, G. M., \& Wurst, S. A. Peripheral and central processes in visual persistence. Paper presented at the annual meeting of the Psychonomic Society, Minneapolis, November 1982.

\section{REFERENCES}

Bowling, A., \& Lovegrove, W. The effect of stimulus duration on the persistence of gratings. Perception \& Psychophysics, 1980, 27, 574-578.

Bowling, A., \& Lovegrove, W. Iconic memory: Fallacies persist (?). Percpetion \& Psychophysics, 1982, 31, 194-198.

Bryden, M. P. Tachistoscopic recognition, handedness, and cerebral dominance. Neuropsychologia, 1965, 3, 1-8.

Conen, G. Components of the laterality effect in letter recognition: Asymmetries in iconic storage. Quarterly Journal of Experimental Psychology, 1976, 28, 105-114.

Coltheart, M. Iconic memory and visible persistence. Perception \& Psychophysics, 1980, 27, 183-228.

Di LoLLo, V. Temporal integration in visual memory. Journal of Experimental Psychology: General, 1980, 109, $75-97$.

Di Lolso, V. Hemispheric symmetry in the duration of visible persistence. Perception \& Psychophysics, 1981, 29, 21-25.

DI Lotto, V., \& Woods, E. Duration of visible persistence in relation to range of spatial frequencies. Journal of Experimental 
Psychology: Human Perception and Performance, 1981, 7, 754-769.

Fontenot, D. J. Visual field differences in the recognition of verbal and non-verbal stimuli in man. Journal of Comparative and Physiological Psychology, 1973, 85, 564-569.

Fukuda, Y., \& Stone, J. Retinal distribution and central projections of $\mathbf{X}-, \mathbf{Y}-$, and $\mathbf{W}$ - cells of the cat's retina. Journal of Neurophysiology, 1974, 37, 749-772.

Harwe rth, R. S., \& Boltz, R. L., \& Smith, E. L., III. Psychophysical evidence for sustained and transient channels in the monkey visual system. Vision Research, 1980, 20, 15-22.

Hawkins, H. L., \& Shulman, G. L. Two definitions of persistence in visual perception. Perception \& Psychophysics, 1979, 25, 348-350.

Long, G. M. Comment on Hawkins and Shulman's Type I and Type II visual persistence. Perception \& Psychophysics, 1979, 26, $412-414$.

Long, G. M. Iconic memory: A review and critique of the study of short-term visual storage. Psychological Bulletin, 1980, 88, 785-820.

Lona, G. M. Persisting problems in persistence: A response to Bowling and Lovegrove. Perception \& Psychophysics, 1982, 32, 192-194.

Long, G. M., \& Benton, R. J. The case for peripheral persistence: Effects of target and background luminance on a partialreport task. Journal of Experimental Psychology: Human Perception and Performance, 1982, 8, 383-391.

Lona, G. M., \& McCArthy, P. R. Target energy effects on Type 1 and Type 2 visual persistence. Bulletin of the Psychonomic Society, 1982, 19, 219-221. (a)

Lona, G. M., \& MCCARThY, P. R. Rod persistence on a partialreport task with scotopic and photopic backgrounds. American Journal of Psychology, 1982, 95, 309-322. (b)

Marzi, C. A., DiStefano, M., Tassinari, G., \& Crea, F. Iconic storage in the two hemispheres. Journal of Experimental
Psychology: Human Perception and Performance, 1979, 5, 31-41.

Meyer, G. E., \& Maguire, W. M. Spatial frequency and the mediation of short-term visual storage. Science, 1977, 198, 524-525.

MEYer, G. E., \& Maguire, W. M. Effects of spatial-frequency specific adaptation and target duration on visual persistence. Journal of Experimental Psychology: Human Perception and Performance, 1981, 7, 151-156.

Polzella, D. J., DaPolito, F., \& Hinsman, M. C. Cerebral asymmetry in time perception. Perception \& Psychophysics, 1977, 21, 187-192.

REGAN, D. Visual information channeling in normal and disordered vision. Psychological Review, 1982, 89, 407-444.

Seraent, J. The cerebral balance of power: Confrontation or cooperation? Journal of Experimental Psychology: Human Perception and Performance, 1982, 8, 253-272. (a)

Sengent, J. Theoretical and methodological consequences of variations in exposure duration in visual laterality studies. Perception \& Psychophysics, 1982, 31, 451-461. (b)

Simion, F., Bagnara, S., Bisiachi, P., Roncato, S., \& UmiLtA, C. Laterality effects, levels of processing and stimulus properties. Journal of Experimental Psychology: Human Perception and Performance, 1980, 6, 184-195.

Umilta, C., Bagnara, S., \& Simion, F. Laterality effects for simple and complex geometrical figures and nonsense patterns. Neuropsychologia, 1978, 16, 43-39.

White, M. J. Hemispheric asymmetries in tachistoscopic information processing. British Journal of Psychology, 1972, 63, 497-508.

Williamson, S. J., Kaufman, L., \& Brenner, D. Latency of the neuromagnetic response of the human visual cortex.Vision Research, 1978, 18, 107-110.

(Manuscript received September 29, 1982; accepted for publication November $5,1982$. 